\title{
Toulouse « Tolosa »
}

Ancien hôpital Larrey

\section{(2) OpenEdition \\ Journals}

Édition électronique

URL : http://journals.openedition.org/adlfi/10217

ISSN : 2114-0502

Éditeur

Ministère de la culture

\section{Référence électronique}

«Toulouse «Tolosa » , ADLFI. Archéologie de la France - Informations [En ligne], Midi-Pyrénées, mis en ligne le 01 mars 1997, consulté le 30 avril 2019. URL : http://journals.openedition.org/adlfi/10217

Ce document a été généré automatiquement le 30 avril 2019.

(c) Ministère de la Culture et de la Communication, CNRS 


\title{
Toulouse « Tolosa »
}

\author{
Ancien hôpital Larrey
}

\author{
Date de l'opération : 1990 (SU) ; 1988 - 1989 (SP) \\ Inventeur(s) : Filippo Raphaël de ; Schaad Daniel
}

1 Dans un secteur situé à l'extrême nord-ouest de la ville antique [ (Fig. $\mathrm{n}^{\circ} 1$ : Localisation des fouilles effectuées dans la ville): plan général, site $\mathrm{M}$ ], une importante fouille de sauvetage conduite par Raphaël de Filippo s'est déroulée sur une superficie de $5000 \mathrm{~m}^{2}$, en préalable à la réalisation d'un grand ensemble immobilier. Une deuxième opération a été menée, en 1990, sur une surface de $500 \mathrm{~m}^{2}$ cette fois, sous la responsabilité de Daniel Schaad, en prévision des travaux d'excavation de l'auditorium du nouveau conservatoire de musique de Toulouse (Fig. $n^{\circ} 2$ : Plan d'ensemble des vestiges antiques mis au jour : enceinte gallo-romaine du début du I ${ }^{\mathrm{er}} \mathrm{S}$. après J.-C. et bâtiments de la Basse Antiquité $\left(\mathrm{V}^{\mathrm{e}} \mathrm{s}\right.$.). Coupe stratigraphique au droit du bâtiment tardif $\mathrm{du} \mathrm{V}{ }^{\mathrm{e}} \mathrm{s}$. parallèle à l'enceinte. ).

2 Aux côtés d'indices ponctuels d'occupation protohistorique, de bâtiments et de nombreuses fosses-dépotoirs datés du bas Moyen Âge, les intérêts majeurs de ces interventions concernent le rempart du Haut-Empire, un atelier de potier du IV s., et un édifice public monumental $\mathrm{du}^{\mathrm{e}} \mathrm{s}$. Nous évoquerons les différents aspects de ce continuum selon leur progression chronologique.

3 Pour la Protohistoire, les structures rencontrées de manière résiduelle sont des trous de poteau de petit diamètre et des fossés qui n'autorisent pas de reconstitution architecturale. Elles sont associées à du mobilier céramique aux formes et décors typiques de la fin du Bronze final et du Premier Âge du fer.

4 L'occupation antique du secteur se caractérise avant tout par la présence du rempart, retrouvé ici en excellent état de conservation (Fig. $\mathrm{n}^{\circ} 3$ : Fondations du premier projet et élévation du rempart). La fouille de 1988 a permis le dégagement de l'intégralité d'une courtine et de deux tours rondes, sur un tronçon de plus de $51 \mathrm{~m}$ de long, pour une élévation de 6,50 $\mathrm{m}$ de haut. Les deux tours sont identiques, de forme circulaire pour un diamètre intérieur de 7,60 $\mathrm{m}$ et extérieur de $10 \mathrm{~m}$, et débordent des deux tiers du côté 
situé extra muros. Leur épaisseur $(1,20 \mathrm{~m})$ est égale à la moitié de celle de la courtine $(2,40 \mathrm{~m})$.

5 L'examen architectonique du rempart révèle un parement de base d'une hauteur de 1,40 m construit en opus vittatum de moellons calcaires, rythmé aux deux tiers de sa hauteur par trois assises de briques qui traversent toute l'épaisseur du mur. Il prend appui sur un puissant béton de fondation en gros galets de Garonne coulé dans une tranchée au fond plat profonde de près d'un mètre. Au-dessus de cette base en pierre calcaire sont conservés 5,10 m de hauteur de mur, dotés d'un parement exclusivement constitué de briques, qui représentent, probablement, la totalité de l'élévation originelle à l'exception du couronnement. La structure interne du bâti présente des murettes de briques disposées en quinconce, formant des caissons transversaux entre lesquels est coulé un mortier de galets d'une grande dureté. La fouille a également mis au jour les fondations d'une tour circulaire et d'un tronçon de courtine long de $10 \mathrm{~m}$, témoins d'un tracé du rempart initialement prévu, puis abandonné encours de réalisation.

Les indices de datation recueillis sur le terrain sont particulièrement intéressants: l'examen du mobilier découvert dans le mortier des fondations comme dans les niveaux de travail contemporains de l'édification montre que le chantier de construction de ce tronçon de l'enceinte a fonctionné entre 30 et 50 après J.-C. Les analyses archéomagnétiques réalisées concluent à une fourchette allant de 0 à 30 après J.-C. pour la base de la muraille, de 0 à 40 pour le sommet.

Quelques dizaines de mètres plus loin vers l'est, le sauvetage urgent conduit par Daniel Schaad a, pour sa part, permis le dégagement de la paroi interne d'un tronçon de courtine long de $24 \mathrm{~m}$, et de l'arrière d'une tour à talon qui ne figurait pas sur les documents cadastraux anciens et modernes. Le talon fait une saillie de 1,50 m par rapport au rempart ; une porte large de 2,88 m est aménagée dans son axe. La face interne du mur a été retrouvée très abîmée, les moellons calcaires du parement de base ayant été à $85 \%$ récupérés.

Une maison antique de plan rectangulaire a été retrouvée à $3,50 \mathrm{~m}$ de distance du rempart : divisée en deux pièces selon une partition deux tiers-un tiers, dotée de trois niveaux de sol en terre battue successifs et d'un four, elle a été édifiée vers le milieu du I ${ }^{\text {er }}$ s. après J.-C. sur une couche de limon stérile qu'entaille la fondation de l'enceinte. Les indices d'implantation dans la seconde moitié $d u \mathrm{I}^{\mathrm{er}} \mathrm{s}$. d'un habitat le long de la fortification, sous la forme de quelques murs en angle droit et d'un sol de tuileau, avaient également été relevés dans la parcelle fouillée par Raphaël de Filippo. Un habitat s'est donc développé à proximitéi mmédiate du rempart peu de temps après l'achèvement de celui-ci, pour être apparemment abandonné dans les premières décennies du second siècle. Un large espace ouvert, formant une sorte d'esplanade, est établi à la fin du $\mathrm{III}^{\mathrm{e}} \mathrm{s}$., au plus tôt.

9 Un atelier de potier lui succède, matérialisé par un canal de chauffe associé à une série de diverticules qui alimentaient probablement les chambres de chauffe des fours situés hors emprise du chantier. Un important dépotoir a également été fouillé, révélant les rebuts d'une production céramique variée, de couleur orangée, à la surface engobée ou lissée et au décor estampé : cruches, jattes, oules, mortiers, écuelles et lampes à huile, dont une de type très particulier qui possède une anse en forme de protomé de cheval. Les lots de monnaies associés à un second dépotoir montrent que l'atelier a dû être en activité pendant toute la seconde moitié du IV $\mathrm{s}$. au moins. 

édifice monumental de très grandes dimensions, adossé sur son flanc nord au rempart. Son plan, qui n'a pu être exhaustivement dégagé lors des fouilles de 1988, présente, selon une parfaite symétrie, deux grandes exèdres opposées, de longs couloirs latéraux contrefortés régulièrement par des piliers, un vestibule central et une entrée occidentale (Fig. ${ }^{\circ} 4$ : Vue d'ensemble de l'édifice à exèdres du IV ${ }^{\mathrm{e}} \mathrm{s}$. après J.-C. ).

De l'axe de symétrie formé par l'entrée occidentale et jusqu'au rempart antique qui ferme le bâtiment au nord, la façade ouest se développe sur $37 \mathrm{~m}$, la façade est sur $45 \mathrm{~m}$. La longueur totale du monument doit donc être estimée entre $74 \mathrm{~m}$ et $90 \mathrm{~m}$ selon la manière, non visible dans l'emprise de la fouille, dont il se termine au sud.

Les fouilles complémentaires de 1990 ont révélé en bordure du rempart et parallèle à celui-ci cette fois, une vaste salle mesurant $15,80 \mathrm{~m}$ de large et plus de $33 \mathrm{~m}$ de long, appartenant à une autre aile de ce grand ensemble architectural.

13 Largement détruits par une récupération systématique des matériaux de construction et par le bâti médiéval, les murs formant l'élévation du monument sont constitués d'assises alternées irrégulièrement de galets de Garonne et de fragments de briques et de tuiles à rebords. Ils reposent sur une fondation profonde de $0,80 \mathrm{~m}$ en moyenne, formée d'une large semelle de petits galets liés au mortier qui couvre plusieurs lits de gros galets séparés entre eux par une couche de gravier.

14 Les sols intérieurs et extérieurs ont pratiquement tous disparu; les rares témoins subsistants sont formés d'une couche de mortier de chaux coulé sur un radier de graviers. Daniel Schaad précise que la présence de tesselles de mosaïque dans le niveau de destruction qui recouvre le sol pourrait suggérer que la couche de mortier aurait correspondu au nucleus d'un décor mosaïqué dont il ne subsisterait malheureusement aucun vestige en place. Raphaël de Filippo a pu observer quelques restes d'enduits muraux intérieurs.

15 Un terminus post quem pour la construction de ce monument est fourni par le mobilier recueilli dans les remblais et les murs des fondations (monnaies fin IV ${ }^{\mathrm{e}} \mathrm{s}$. et ornement de ceinture en bronze du premier quart $d u V^{e} s$.). Les analyses ${ }^{14} \mathrm{C}$ faites sur le bois brûlé provenant de la couverture donnent une fourchette allant de 350 à 550 . En l'absence de toute couche d'occupation significative, l'hypothèse la plus vraisemblable plaide pour une construction courant $\mathrm{V}^{\mathrm{e}} \mathrm{s}$. de cet édifice public à caractère monumental dont l'identification précise reste à déterminer.

16 Après une phase de récupération des matériaux de construction que le mobilier retrouvé dans les tranchées creusées à cet effet, situe chronologiquement entre le $\mathrm{X}^{\mathrm{e}} \mathrm{s}$. et le XII ${ }^{\mathrm{e}} \mathrm{s}$., c'est à la fin du XIII' $s$. et au XIV ${ }^{\mathrm{e}} \mathrm{s}$. que ce quartier, ayant appartenu au moulon $5 \mathrm{du}$ capitoulat de la Daurade, subit une nouvelle organisation.

17 La partie nord du secteur, explorée en 1988-1989, est laissée libre de toute construction et utilisée pour le creusement d'une centaine de fosses-dépotoirs, dont la fouille du comblement a livré une importante quantité de mobilier:céramiques, verreries, faune, graines, pépins et coquillages.

$18 \mathrm{Au}$ sud est implanté un bâtiment composé de plusieurs pièces réaménagé à plusieurs reprises entre le XIII $s$. et le début duXVIes., faisant successivement fonction d'habitation, de dépotoir et d'entrepôt. 
La zone fouillée en 1990 a révélé partiellement le plan de deux grandes maisons appuyées au rempart et séparées par une ruelle.

Les sœurs de Notre-Dame achètent l'ensemble des terrains concernés, en 1634, pour y faire construire leur couvent,provoquant la destruction, courant XVII ${ }^{e}$., de toutes les habitations médiévales.

\section{ANNEXES}

Fig. $\mathrm{n}^{\circ} 1$ : Localisation des fouilles effectuées dans la ville

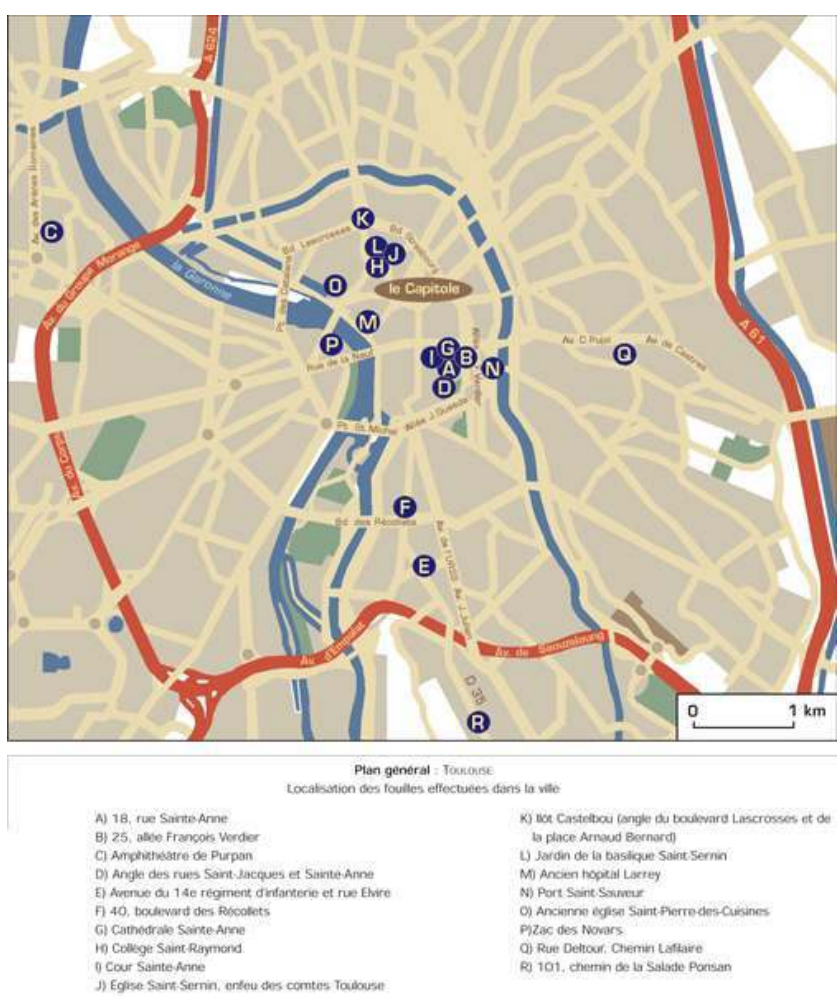

GI 1997 ; CNRS Éditions 1998 (1994) 
Fig. $n^{\circ} 2$ : Plan d'ensemble des vestiges antiques mis au jour : enceinte gallo-romaine du début du I ers. après $\mathrm{J}$.-C. et bâtiments de la Basse Antiquité $\left(\mathrm{V}^{\mathrm{e}} \mathrm{s}\right.$.). Coupe stratigraphique au droit du bâtiment tardif du $\mathrm{V}^{\mathrm{e}} \mathrm{s}$. parallèle à l'enceinte.

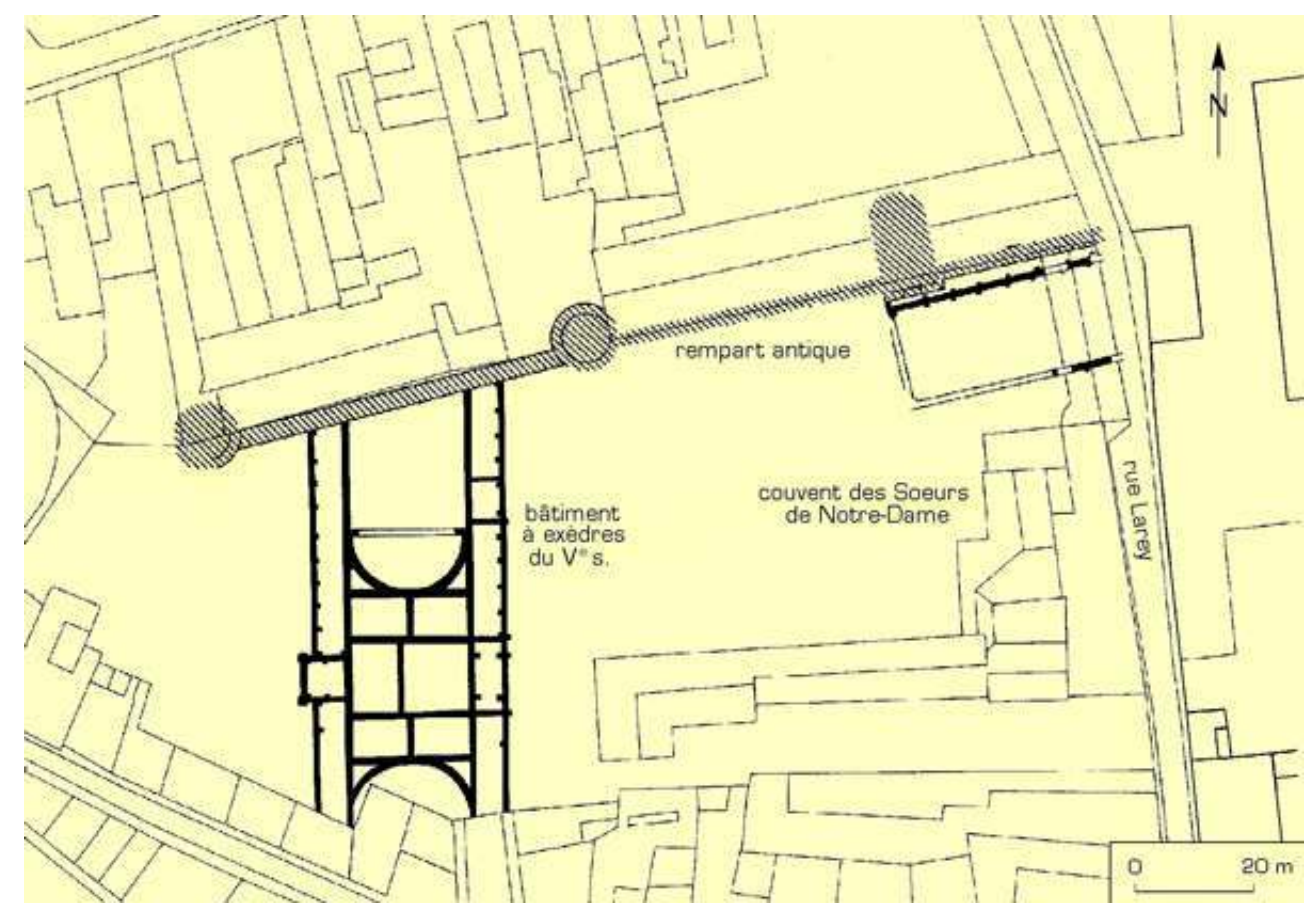

Auteur(s) : Filippo, Raphaël de ; Schaad, Daniel. Crédits : Gi 1997 ; CNRS Éditions 1998 (1990)

Fig. $n^{\circ} 3$ : Fondations du premier projet et élévation du rempart

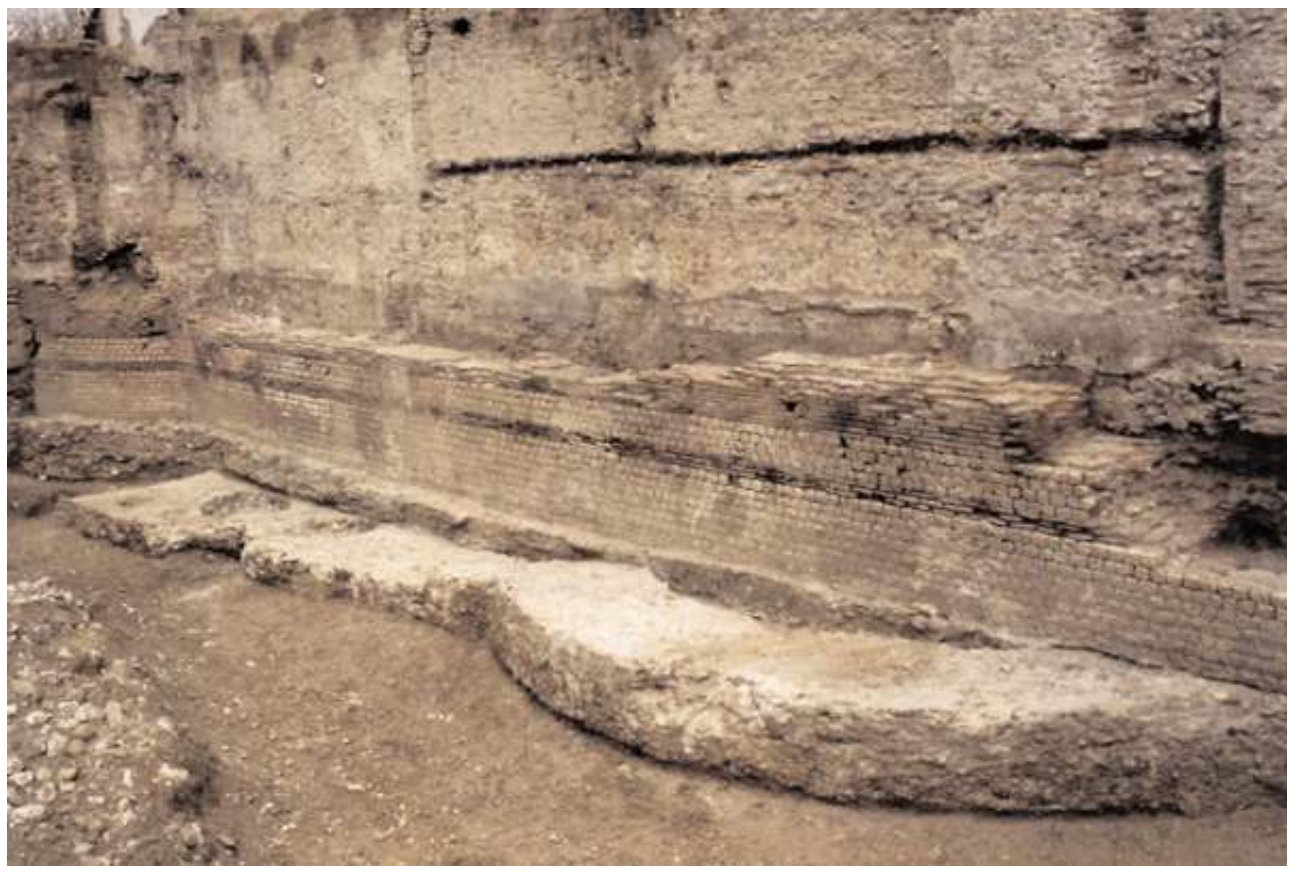

Gl 1997 ; CNRS Éditions 1998 (1990) 
Fig. $n^{\circ} 4$ : Vue d'ensemble de l'édifice à exèdres du IV ês. après J.-C.

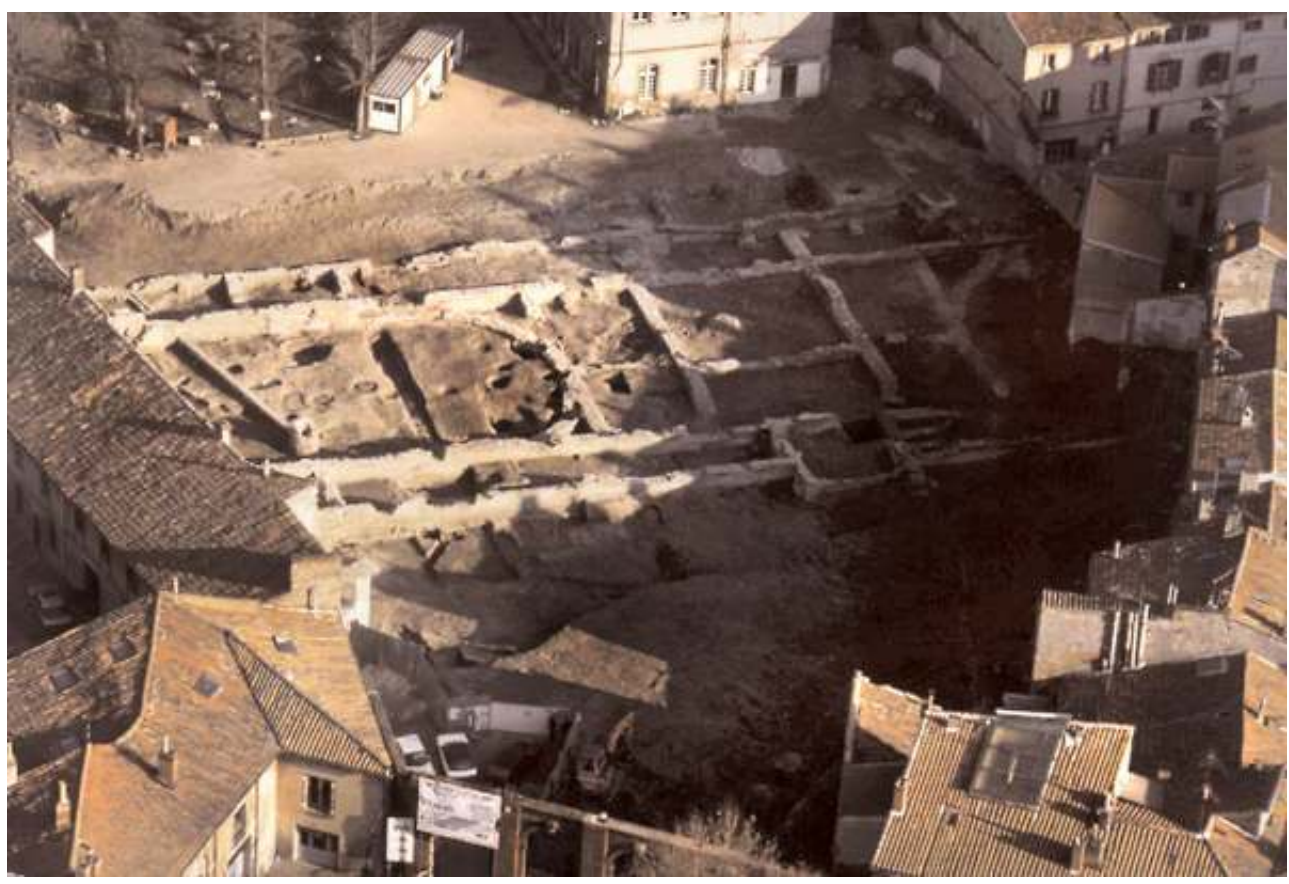

Auteur(s) : Filippo, Raphaël de. Crédits : GI 1997 ; CNRS Éditions 1998 (1990)

INDEX

operation sauvetage urgent (SU), sauvetage programmé (SP)

Index chronologique : Antiquité tardive, Bronze final, Empire romain, ép médiévale, Premier âge du Fer

Index géographique : Midi-Pyrénées, Haute-Garonne, Toulouse 